\title{
Erste SAP Mobile Engine Österreichs im Einsatz bei ONE
}

\author{
Der Wiener SAP-Berater CNT Management Consulting hat auf Basis der SAP Mobile Engine eine \\ mobile Anwendung für den Einsatz bei ONE implementiert. Die Applikation sorgt dafür, dass ge- \\ schäftliche Transaktionen und Servicemeldungen mobil über einen Pocket-PC abgewickelt werden.
}

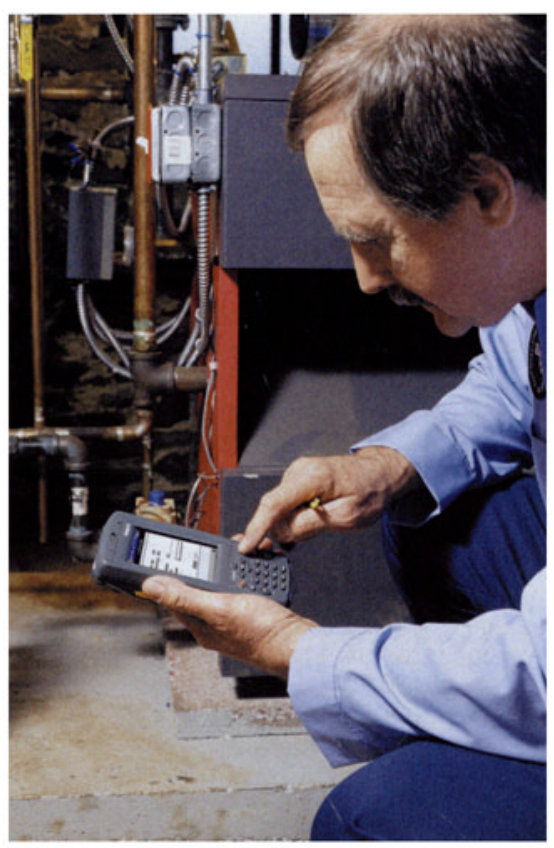

Bei Defekt einer Sendeanlage können die Servicetechniker verschiedenste Transaktionen vor Ort über einen Pocket-PC abwickeln

Die Servicetechniker können nunmehr an jedem Einsatzort Materialbuchungen vornehmen, Reparaturen an Sendeanlagen selbständig digital erfassen, nach Beendigung der Arbeiten mit dem zentralen ERP-System synchronisieren und somit die Instandhaltung zeitaktuell rückmelden. Das garantiert einfache Abwicklung und aktuellen Datenabgleich zwischen ONE und der Alcatel Austria AG, zu der ONE mit Anfang 2004 die Bereiche Netzwerkaufbau und -betrieb ausgelagert hat.

\section{Logistik und Instandhaltung}

Nach Kanada, Schweden und Deutschland wird das Mobile Business-Produkt SAP Mobile Engine nun erstmals in Österreich eingesetzt. Abgesehen von Bell Canada ist es vor allem in Europa in Verwendung, wie bei den Energieunternehmen Sydkraft $A B$ (Schweden) und Wesertal GmbH (E-on Deutschland). Bei ONE unterstützt die SAP Mobile Engine die Datenverarbeitung in der Logistik und Instandhaltung. Mit einem outdoorfähi-

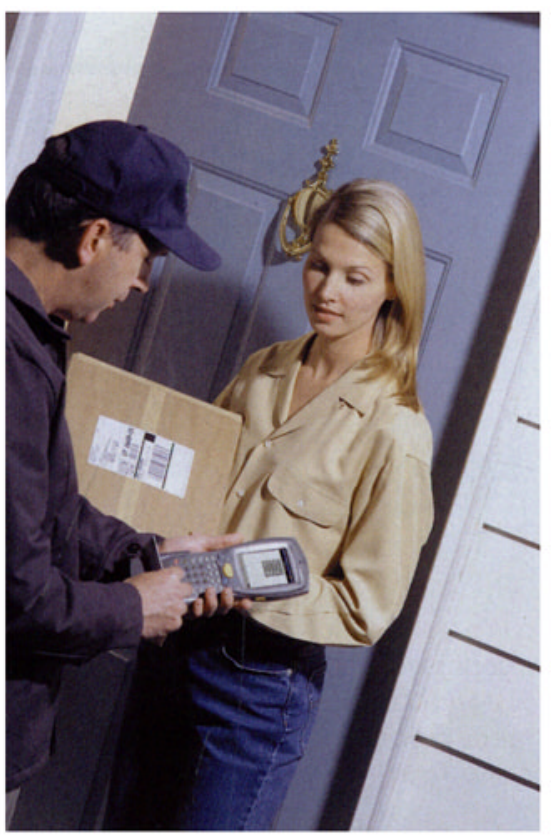

Eine Verbindung zur Mobile Engine kann über GPRS direkt beim Kunden aufgebaut werden

gen, stoßsicheren Pocket-PC inklusive eigens konfigurierter SIM-Karte kann der Servicetechniker von unterwegs über GPRS eine Verbindung zur Mobile Engine aufbauen.

\section{Flexibles Service}

„Die Mobile Engine ist eine sehr praktische und flexible Lösung für unsere Servicetechniker", informiert Christian Ivad, Leiter Business Support Services Development von ONE. "Handschriftliche Aufzeichnungen und Ausfüllen von Papierformularen bei Instandhaltungsaufträgen fallen jetzt weg. Außerdem müssen keine nachträglichen Materialbuchungen mehr gemacht oder handschriftliche Notizen durch das Back-Office erfasst werden. So sind die Daten einer Sendeanlage und Instandhaltungsaufträge auf Knopfdruck ständig aktuell." Für die Logistik sind die Warenbewegungen übersichtlich und nachvollziehbar, der Einkauf kann sich besser orientieren und schneller reagieren, welche Materialien nachbestellt werden müssen.

\section{Die Mobile Engine in der Praxis}

Praktisch funktioniert das System so: Der Servicetechniker muss wegen eines defekten Gerätes eine bestimmte Sendeanlage überprüfen. Zu diesem Zweck lädt er sich die technischen Daten dieser Sendeanlage aus dem zentralen ERP-System (SAP R/3) auf seinen Pocket-PC und eröffnet einen Instandhaltungsauftrag. Dann fährt er zur Sendeanlage, ersetzt das defekte Gerät durch ein neues, erfasst währenddessen die Barcodes beider Geräte mittels eingebauten Scanners im PDA und schließt den Auftrag ab.

Bei nächster Gelegenheit baut der Servicetechniker eine GPRS-Verbindung mit dem zentralen ERP-System auf und synchronisiert die Daten über den Mobile Engine Server. Die Materialbuchungen erfolgen in diesem Augenblick, und die Daten über die Sendeanlage und die Materialbewegungen sind sofort aktualisiert.

\section{Komponenten und Systeme}

Die SAP Mobile Engine täuft serverseitig auf einem SAP Web Application Server 6.20 (WAS) und clientseitig wird das plattformunabhängige Mobile Engine Framework benötigt. Darauf aufbauend entwickelte CNT gemeinsam mit der Firma Anecon die Applikation Netzwerklogistik (NWL).

Die Server-Komponenten der Mobile Engine laufen auf der JAVA 2 Enterprise Edition (J2EE) Engine, die mit der ClientKomponente kommuniziert. Der JAVAConnector wird als Verbindung zur Teilkomponente SAP im WAS benötigt, damit die Clients und das SAP CoreSystem, in dem sich die Businesslogik befindet, kommunizieren können. Für die Kommunikation der Systeme sind der Personal Digital Assistent (PDA), der Web Application Server (WAS) und das SAP Core-System R/3 Release 4.6b erforderlich.

Informationen: CNT Management Consulting $\mathrm{GmbH}$, Günter Buchta, Lassallestraße $7 \mathrm{~b}$, A-1020 Wien, Telefon +43 (1) 21642 68-0, E-Mail: g.buchta@cnt-online.at,

Internet: www.cnt-online.at 